\title{
A Splice Variant of the $\mathrm{C}_{2} \mathrm{H}_{2}$-Type Zinc Finger Protein, ZNF268s, Regulates NF-kB Activation by TNF $-\alpha$
}

Jung Nyeo Chun ${ }^{1,4}$, In Sung Song ${ }^{1,4}$, Dong-Hoon Kang ${ }^{1}$, Hye Jin Song ${ }^{1}$, Hye In Kim ${ }^{1}$, Ja Won Suh ${ }^{1}$, Kong Ju Lee ${ }^{1,2}$, Jaesang Kim ${ }^{1,3}$, and Sang Won Kang ${ }^{1,3,4}$

${ }^{1}$ Division of Life and Pharmaceutical Sciences and Center for Cell Signaling Drug Discovery Research, Ewha Womans University, Seoul 127750 , Korea, ${ }^{2}$ College of Pharmacy, Ewha Womans University, Seoul 127-750, Korea, ${ }^{3}$ Department of Life Science, Ewha Womans University, Seoul 127-750, Korea, ${ }^{4}$ These authors contributed equally to this work.

Received January 20, 2008; accepted January 30, 2008; published online August 4, 2008

In the article by Chun et al. that appears in the journal (Mol. Cells 26, 175-180, August 31, 2008), AUTHOR NAMES and ACKNOWLEDGMENTS should be revised as written below.

Jung Nyeo Chun ${ }^{1,4}$, In Sung Song ${ }^{1,4}$, Dong-Hoon Kang ${ }^{1}$, Hye Jin Song ${ }^{1}$, Hye In Kim ${ }^{1}$, Jawon Seo ${ }^{1}$, Kong-Joo Lee ${ }^{1,2}$, Jaesang Kim ${ }^{1,3}$, and Sang Won Kang ${ }^{1,3}$

${ }^{1}$ Division of Life and Pharmaceutical Sciences and Center for Cell Signaling Drug Discovery Research, Ewha Womans University, Seoul 127750 , Korea, ${ }^{2}$ College of Pharmacy, Ewha Womans University, Seoul 127-750, Korea, ${ }^{3}$ Department of Life Science, Ewha Womans University, Seoul $127-750$, Korea, ${ }^{4}$ These authors contributed equally to this work.

Received January 20, 2008; accepted January 30, 2008; published online August 4, 2008

\section{ACKNOWLEDGMENTS}

This work was supported by the Korea Research Foundation Grant (KRF-2004-015-C00439), KOSEF through the Center for Cell Signaling \& Drug Discovery Research (CCS \& DDR, R15-2006-020) at Ewha Womans University, and 21C Frontier Functional Proteomics Project from Ministry of Science \& Technology (FPR08-B1-190). This work was also supported by the second phase of Brain Korea 21 project (J. N. Chun, H. J. Song). 